\title{
Application of 2,3-Dichloro-5,6-Dicyano-P-Benzoquinone (DDQ) For Spectrophotometric Determination of Caroverine In Pharmaceutical Formulations
}

\author{
Asad Raza* and Tariq Mahmood Ansari \\ Division of Analytical Chemistry, Institute of Chemical Sciences Bahauddin Zakariya University Multan 60800,
} Pakistan

Received: June 08, 2017; Accepted: August 18, 2017; Published: August 31, 2017

*Corresponding author: Asad Raza, Division of Analytical Chemistry, Institute of Chemical Sciences Bahauddin Zakariya University Multan 60800, Pakistan, E-mail: asadbzu@gmail.com

\begin{abstract}
A new, simple, sensitive and rapid spectrophotometric method is developed for the quality control of caroverine in pharmaceutical pure and dosage forms. The method is based on the application of 2,3-dichloro-5,6dicyano-p-benzoquinone (DDQ) as $\pi$-acceptors for the determination of caroverine which act as an n-donor to form a highly colored charge transfer complex with absorption band at $570 \mathrm{~nm}$. The utility of the proposed method is established by the determination of the studied drug in commercial tablets and the results are statistically assessed as per the ICH guidelines. The newly proposed procedure is successfully applied to the determination of caroverine with good recovery and percent ranged from 99.0 to $101 \%$. The association constant and free energy change using Benesie Hildebrand plot is also presented.
\end{abstract}

Key words: Caroverine; Spectrophotometric; Charge transfer complex; 2, 3-dichloro-5, 6-dicyano-p-benzoquinone (DDQ)

\section{Introduction}

A counterfeit medication or a counterfeit drug is a medication or pharmaceutical product which is produced and sold with the intent to deceptively represent its origin, authenticity or effectiveness. A counterfeit drug may contain inappropriate quantities of active ingredients, or none, may be improperly processed within the body (e.g., absorption by the body), may contain ingredients that are not on the label [1]. The concern about the quality of drugs marketed increases every year not only in commercial terms, but also legal and ethical aspects, since the health of patients depends on the quality and effectiveness of these drugs. For this purpose different regulatory authorities around the world are demanding specific and validated analytical methods for the registration of new drugs to ensure their quality. So there is a great interest in developing rapid and efficient analytical methods that provide precise and accurate parameters for the quantitative analysis of drugs in pharmaceutical raw and dosage forms. Caroverine1-(2-diethylaminoethyl)-3-(p-methoxybenzyl)-1,2dihydro-2-quinoxalin-2-on-hydrochloride is chemically derived from isoquinoline, the basic structure of papaverin. It is clinically available in some countries as a spasmolytic drug based on its unspecific $\mathrm{Ca} 2+$ channel blocking activity for more than 40 years. Caroverine is a drug used as a spasmolytic and otoneuroprotective (inner ear protective) agent in some countries. It acts as an N-type calcium channel blocker, competitive AMPA ( $\alpha$-amino-3-hydroxy-5-methyl-4-isoxazolepropionic acid receptor) receptor antagonist, and non-competitive NMDA (N-methyl-D-aspartate receptor) receptor antagonist [2]. It also has potent antioxidant effects [3]. In Pakistan, caroverine is marketed as Saprina Tablets $20 \mathrm{mg}$ (BioPharma, Multan, Pakistan) for oral smooth muscle spasms.

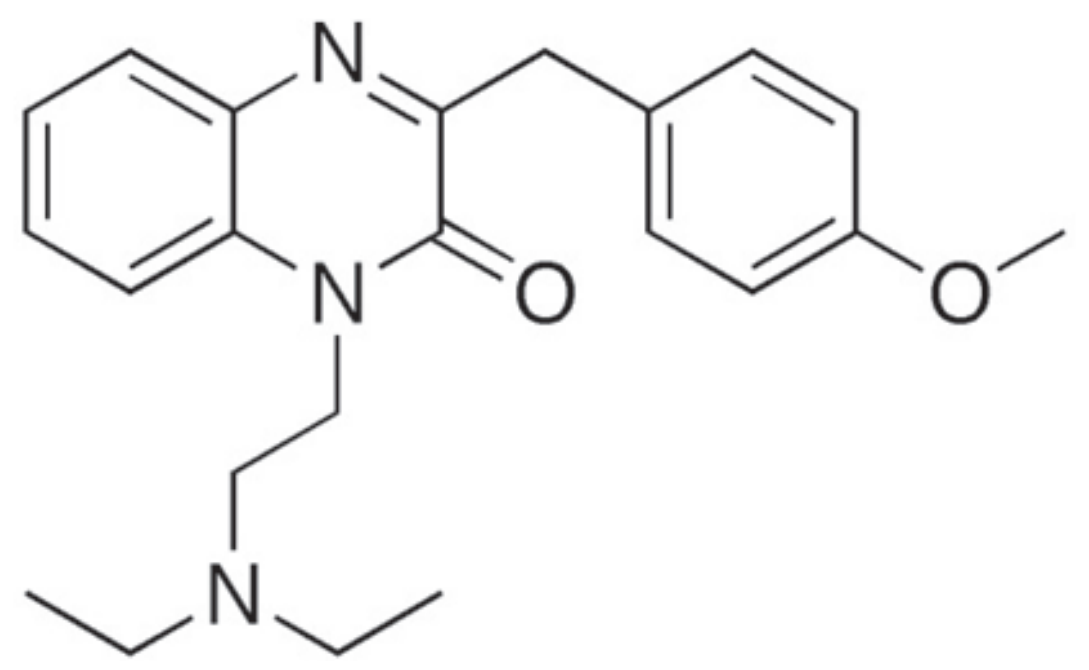

Figure 1: Chemical structure of Caroverine

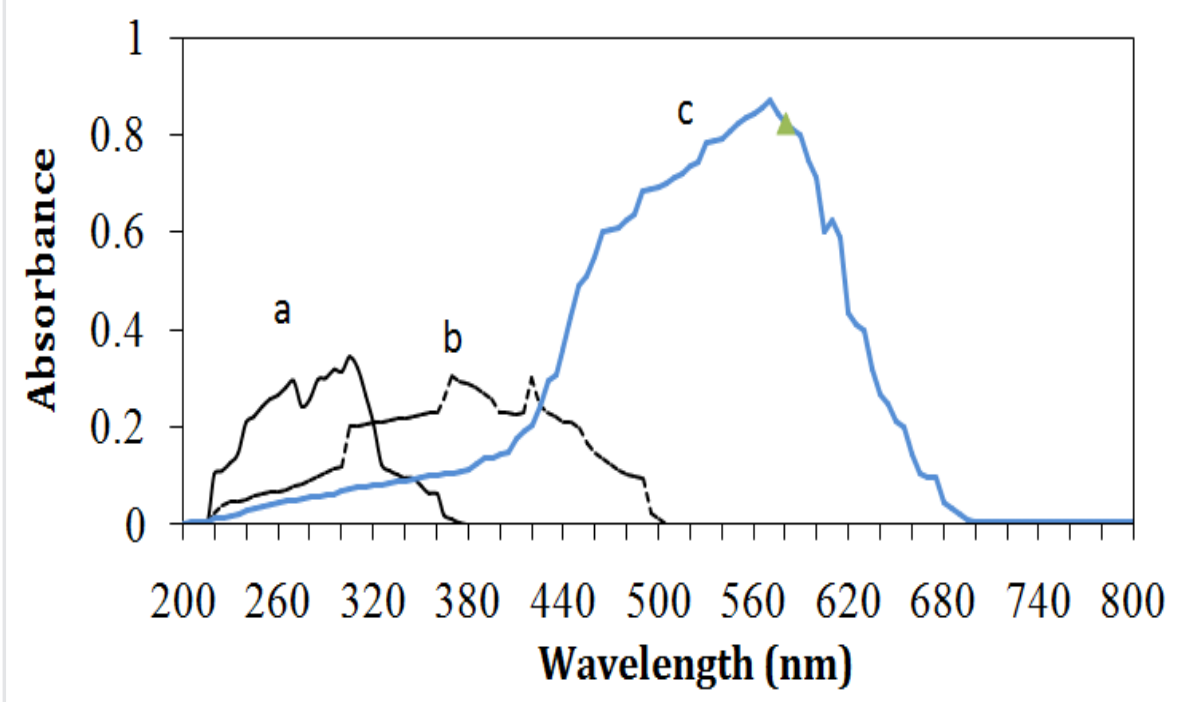

Figure 2: Absorption spectra of (a) caroverine in DMSO, (b) DDQ in methanol, (c) caroverine -DDQ complex in DMSO 


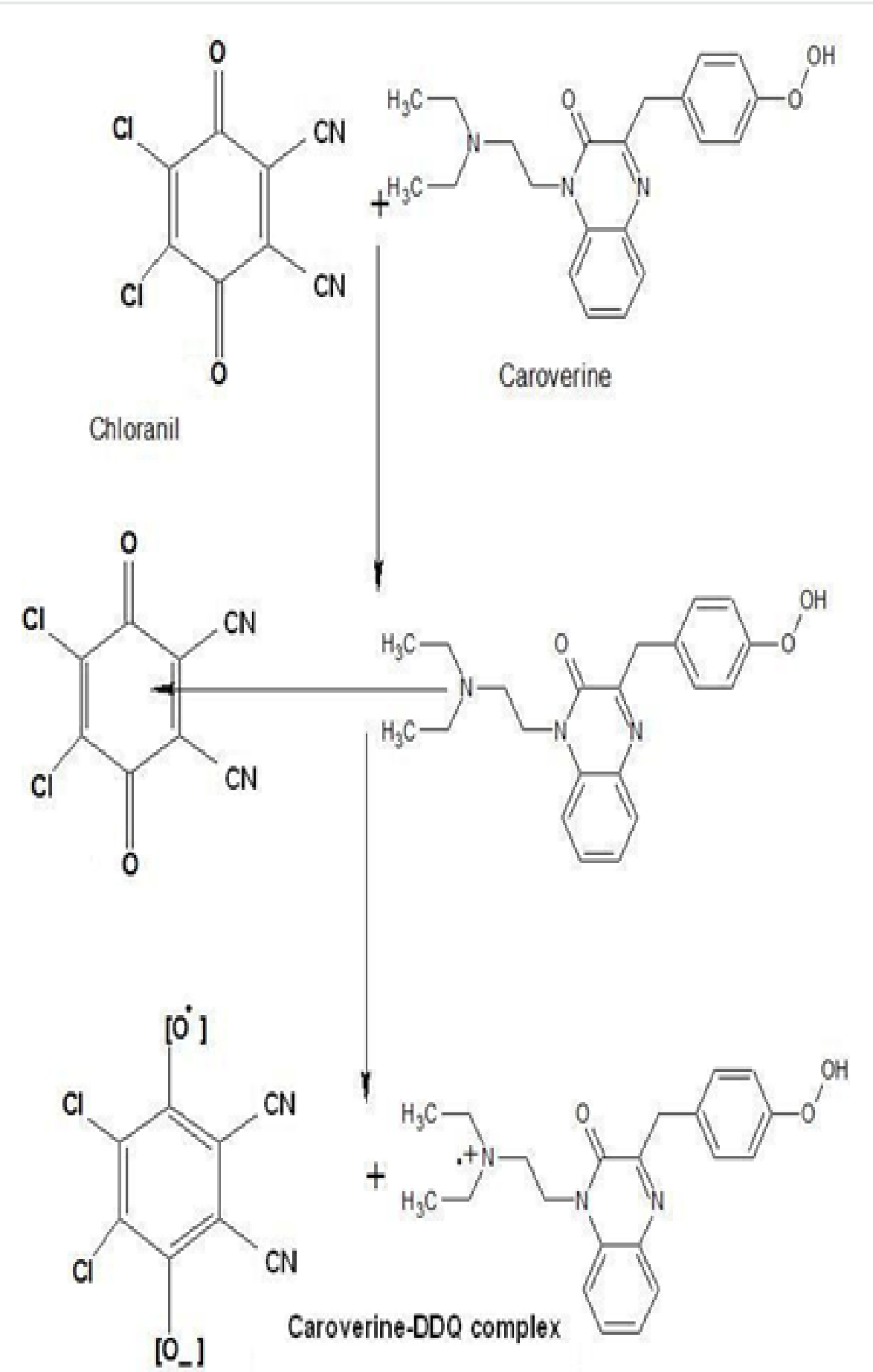

Scheme-1: Reaction of caroverine with DDQ

\section{Optimization of experimental conditions}

In order to establish the optimum reaction conditions suitable for the complexation, various analytical parameters were studied. The effect of each parameter was observed by altering one parameter at a time while keeping others constant. Various analytical solvents were checked like methanol, water, acetonitrile and Dimethy formamide (DMF) and dimethyl sulfoxide (DMSO). But the DMSO was found to be suitable solvent due to high sensitivity and maximum absorbance. Results are shown in Table 1 and Figure 3.

To study the optimum reaction time the absorbance of complex was measured $0,2,5$ and 10 minutes. It was noticed that complete color development was achieved instantaneously at ambient temperature (25 $\pm 2^{\circ} \mathrm{C}$ ) and there was no effect on absorbance at different time interval. The formed color complex was found to be stable for $24 \mathrm{~h}$. To determine the effect of DDQ concentration, the concentration of caroverine was kept constant and the concentration of DDQ solution was changed by varying the ml of stock solution.

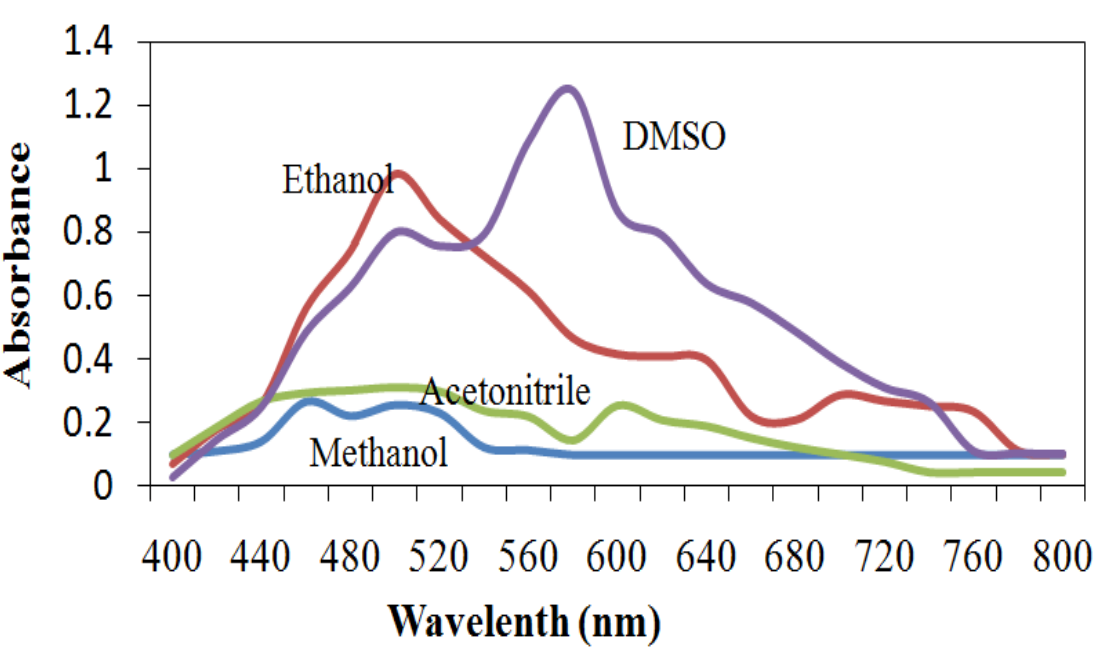

Figure 3: Absorption spectra of reaction mixture of caroverine with DDQ

\begin{tabular}{|c|c|c|c|c|c|c|}
\hline \multicolumn{7}{|c|}{ Table 1: Effect of solvent on the interaction of caroverine with DDQ } \\
\hline Solvent & $\begin{array}{c}\text { Mol. Wt. } \\
\text { (g.mol) }\end{array}$ & $\begin{array}{c}\text { Dielectric } \\
\text { constant }\end{array}$ & Absorbance & Solubility & $\begin{array}{c}\boldsymbol{K} \\
\text { max }\end{array}$ & $\begin{array}{c}\text { M o l a r } \\
\text { absorptivity } \\
\text { L mol }^{-1} \mathbf{S}^{-1}(\epsilon)\end{array}$ \\
\hline Methanol & 32.04 & 32.7 & 0.358 & Partially & 460 & $3.4 \times 103$ \\
\hline Ethanol & 46.06 & 24.6 & 1.19 & Partially & 470 & $4.4 \times 103$ \\
\hline Acetonitrile & 41.05 & 37.5 & 0.145 & Partially & 520 & $4.1 \times 102$ \\
\hline DMSO & 78.13 & 46.7 & 1.54 & Completely & 570 & $1.5 \times 104$ \\
\hline
\end{tabular}

\section{Reagent Concentration}

We found that $0.5 \mathrm{ml}$ is the optimum volume of DDQ solution for complete color development. It wasfound that the increased concentrations of the reagent have no mark effect on the color development. It is clear from the Figure 4.

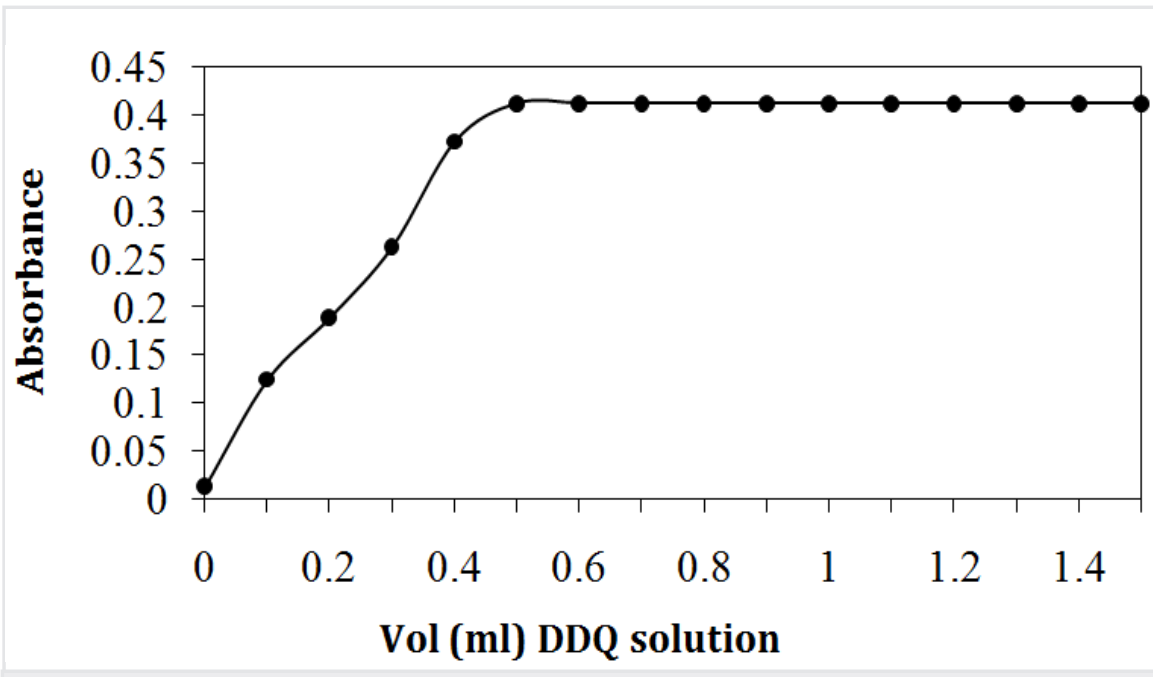

Figure 4: Effect of reagent concentration on color formation

\section{Reaction time}

Following the absorbance of the developed color at different time intervals at ambient temperature $\left(25 \pm 5{ }^{\circ} \mathrm{C}\right)$ it was Concluded that complete color development was attained instantaneously. But for more accuracy and safety we placed the reaction mixture for five minutes at room temperature before taking the absorbance measurement.

\section{Stoichiometric ratio of complex}

The stoichiometric ratio of caroverine and DDQ was established by applying Job's method of continuous variation using equimolar solutions by taking absorbance of complex solutions of different ratios $(0: 10,1: 9$, 
........10:0) (donor: acceptor). The graph was plotted between the mole fractions of drug vs. absorbance. Figure 5 indicates that caroverine interacted with DDQ in stoichiometric ratio of 1:1.

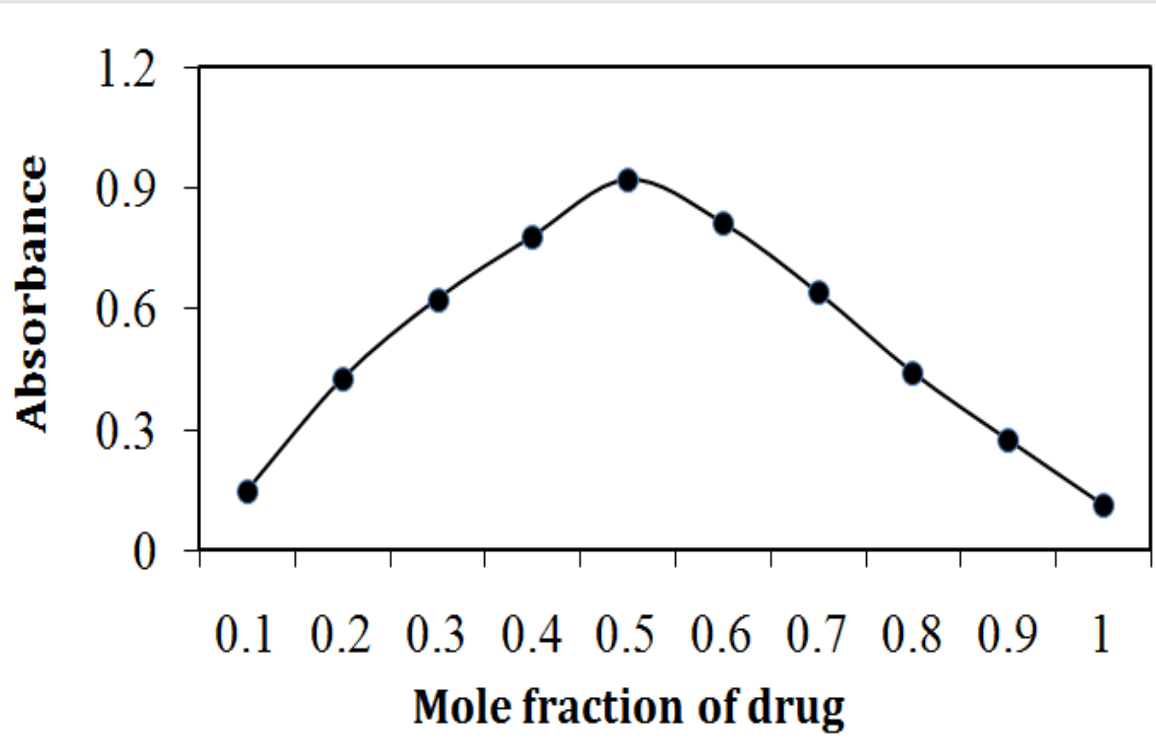

Figure 5: Job's plot of caroverine and DDQ complex

\section{Association constants and standard free energy changes}

The association constant was determined for the interaction of caroverine with DDQ complex using Benesi Hildebrand equation [8].

$$
\frac{C a}{A}=\frac{1}{\varepsilon}+\frac{1}{K C \times \varepsilon} \times \frac{1}{C b}
$$

Where $\mathrm{Ca}$ and $\mathrm{Cb}$ are the concentrations of the acceptor and donor respectively, $\mathrm{A}$ is the absorbance of the complex, $\varepsilon$ is the molar absorptivity of the complex and $\mathrm{Kc}$ is the association constant of the complex. The standard free energy changes of complexation $\left(\Delta \mathrm{G}^{\circ}\right)$ were calculated from the association constants by the following equation [9].

$$
\Delta \mathrm{G}^{\mathrm{o}}=-2.303 \mathrm{R} T \log \mathrm{Kc}
$$

Where $\Delta \mathrm{G}^{\mathrm{o}}$ is the free energy change of the complex $\left(\mathrm{kJ} \mathrm{mol}^{-1}\right)$, $\mathrm{R}$ the gas constant $\left(0.001987 \mathrm{~K} \mathrm{cal} \mathrm{mol}^{-1} \mathrm{deg}^{-1}\right), \mathrm{T}$ the temperature in Kelvin $\left(273+{ }^{\circ} \mathrm{C}\right)$ and $\mathrm{Kc}$ is the association constant of drug- acceptor complex $\left(1 \mathrm{~mol}^{-1}\right)$.

\section{Interference of excipients}

More than $99 \%$ recovery of caroverine was obtained in the presence of possible excipients and other additives in tablet formulations such as microcrystalline cellulose, magnesium stearate, titanium dioxide and lactose. Under the experimental conditions employed, to a known amount of drug (caroverine $10 \mu \mathrm{g} / \mathrm{ml}$ ), excipients in different concentrations are added and analyzed. The results of the recovery analysis are presented in Table 1. Excipients up to the concentrations shown in the Table 1 do not interfere with the assay. In addition recoveries in most cases are $100 \%$.
Table 2: Percent recovery of the caroverine in the presence of possible excipients used in tablet formulation

\begin{tabular}{|c|c|c|}
\hline Excipients & $\begin{array}{c}\text { Amount taken } \\
(\mathbf{m m} / \mathbf{m l})\end{array}$ & $\begin{array}{c}\text { \% Recovery } \pm \text { RSD } \\
\text { (n =5) }\end{array}$ \\
\hline Microcrystalline cellulose & 250 & $100.25 \pm 0.31$ \\
\hline Magnesium stearate & 100 & $100.23 \pm 0.25$ \\
\hline Titanium dioxide & 100 & $100.44 \pm 0.37$ \\
\hline Lactose & 350 & $99.85 \pm 0.43$ \\
\hline
\end{tabular}

\section{Validation of method and applications}

The method was validated as per the ICH [10] in terms of linearity, accuracy and specificity, intra-day and inter-day precision, repeatability of measurement of absorbance. Optical characteristics, statistical data for the regression equation and Benesi Hildebrand equation of the proposed method are given in Table 3. The proposed method was found to give linear calibration curve over the concentration ranges of $2-40 \mu \mathrm{g} / \mathrm{ml}$ with a regression coefficient (r) of 0.99958 , indicating good linearity (Figure 6). Assay was performed in triplicate at different levels. This was repeated with a second instrument, standard and sample preparation on different days. These results show that the proposed method has good repeatability and reproducibility. Also the assay results are unaffected by the presence of excipients, thus establish specificity of the method. The proposed method was applied for the determination of the caroverine in commercial preparations. Three replicate determinations were made. Moreover, to check the validity of the proposed method, the standard addition method was applied by adding pure caroverine to the previously analyzed tablets. The recovery of the drug was calculated by comparing the concentration obtained from the spiked mixtures with those of the pure drug. The results of analysis of commercial dosage forms and the recovery study (standard addition method) of the cited drug (Table 4) suggested that there is no interference from any excipients, which are present in tablets.

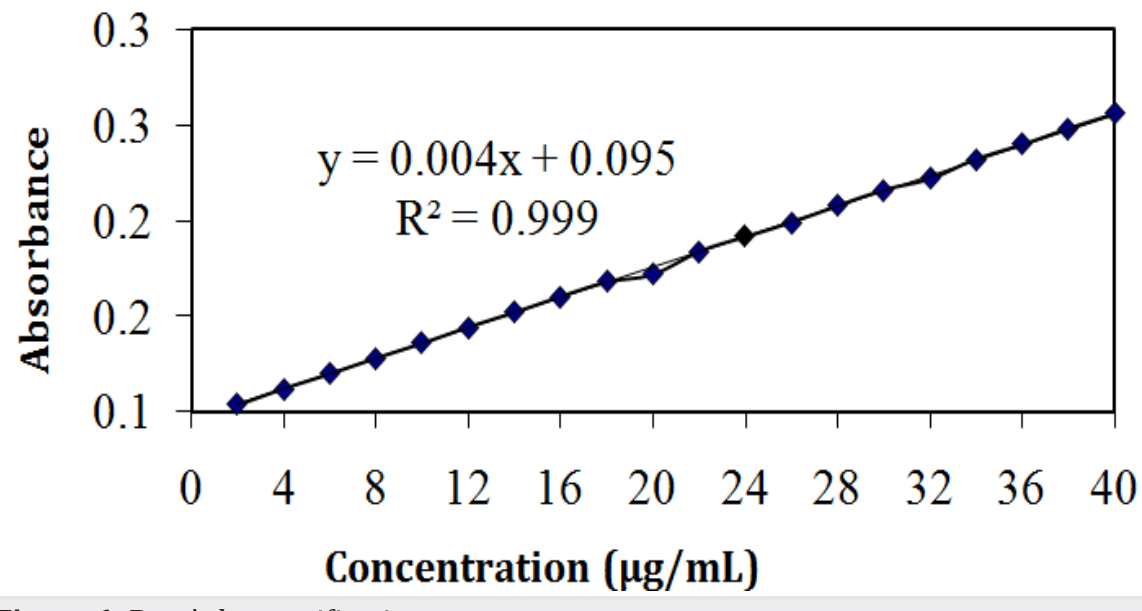

Figure 6: Beer's law verification range

\section{Accuracy and Precision}

Accuracy and precision were investigated by analyzing caroverine tablets (i.e. $20 \mathrm{mg}$ tablet) in three independent replicates on the same day (Intra-day accuracy and precision) and on three consecutive days (Interday accuracy and precision). Intra-day and Inter-day relative standard deviation (RSD) values and also the low RSD values obtained from the analysis of the pharmaceutical tablet formulation (Table 4) indicated good intermediate precision of method. 


\begin{tabular}{|c|c|}
\hline Table 3: Spectral and Benesi-Hildebrand data caroverine-DDQ complex \\
\hline Parameters & Values \\
\hline$\lambda$ max $(\mathrm{nm})$ & 570 \\
\hline Beer's law limits $(\mathrm{mg} / \mathrm{ml})$ & $2-40$ \\
\hline Molar absorptivity $\left(\mathrm{L} \mathrm{mole}-{ }^{-1} \mathrm{~cm}^{-1}\right)$ & $1.5 \times 10^{4}$ \\
\hline Detection limit $\left(\mathrm{mg} / \mathrm{ml}^{-}\right)$ & 1.6 \\
\hline Quantification limit $\left(\mathrm{mg} / \mathrm{ml}^{-}\right)$ & 5.2 \\
\hline Sandle sensitivity $\left(\mathrm{mg} \mathrm{cm}{ }^{-2}\right)$ & $2.3 \times 10^{-2}$ \\
\hline Slope & $3.9 \times 10^{-3}$ \\
\hline Intercept & $9.6 \times 10^{-2}$ \\
\hline Correlation coefficient & 0.9995 \\
\hline Association Constant $(\mathrm{Kc})$ & $1.3 \times 10^{5}$ \\
\hline Standard free energy change $\left(\Delta \mathrm{G}^{\circ}\right)$ & $-7 . .647$ \\
\hline
\end{tabular}

\section{Conclusion}

The reported spectrophotometric method is novel in the sense that none of the analytical method is available in the literature for the quantitative determination of caroverine in pharmaceutical raw and dosage forms. The newly proposed spectrophotometric method is rapid, simple, precise and accurate. The proposed procedure is free from tedious steps like extraction or heating and involves the least number of experimental variables, which is reflected in high precision. An additional advantage of this method is its specificity. Since basic nitrogen is the reaction site, the method is specific to caroverine since none of the excipients normally used in dosage forms contains basic nitrogen. Furthermore, all the analytical reagents are inexpensive, have excellent shelf life, and are easily available in any analytical laboratory. The proposed method can be applied in quality control laboratories for the routine analysis of the caroverine in raw materials and pharmaceutical formulations.

Table 4: Results of recovery study by standard addition method and precision

\begin{tabular}{|c|c|c|c|c|c|c|c|}
\hline \multirow{2}{*}{ Tablets (API) } & $\begin{array}{c}\text { Amount of } \\
\text { drug (mg) in } \\
\text { formulation }\end{array}$ & $\begin{array}{c}\text { Amount } \\
\text { Added } \\
\text { (mg) }\end{array}$ & $\begin{array}{c}\text { Amount } \\
\text { Recovered } \\
\text { (mg) }\end{array}$ & \% Recovery & RSD (\%) & $\begin{array}{c}\text { Precision (Intra } \\
\text { day)* }\end{array}$ & Precision (Inter day)* \\
\hline \multirow{2}{*}{ Sparina Tab } & 20 & 5 & 24.87 & 99.48 & 0.16 & 0.98 \\
\cline { 2 - 8 } Caroverine & 20 & 10 & 29.75 & 99.16 & 0.67 & 1.03 & 0.01 \\
\cline { 2 - 8 } & 20 & 15 & 34.72 & 99.21 & 0.74 & 0.98 \\
\hline *Average of three determinations \\
\cline { 2 - 8 }
\end{tabular}

\section{Acknowledgement}

The authors are grateful to Institute of Chemical Sciences, Bahauddin Zakariya University Multan, Pakistan and BioFine Pharmaceuticals (Pvt.) Ltd., Multan, Pakistan, for providing necessary research facilities to carry out this research.

\section{References}

1. M Davison. Pharmaceutical Anti Counterfeiting: Combating the Real Danger from Fake Drugs. Wiley. 2011;426pp.

2. N Udilova, AV Kozlov, W Bieberschulte, K Frei, K Ehrenberger and H Nohl. The antioxidant activity of caroverine. Biochem Pharmacol. 2003;65(1):59-65.

3. H Nohl, W Bieberschulte, B Dietrich, N Udilova and AV Kozlov. Caroverine, a multifunctional drug with antioxidant functions. BioFactors. 2003;19(1-2):7985.

4. R Foster. Organic Charge Transfer Complexes. Academic Press. 1969;15:387.
5. A Raza and TM Ansari. Development and applications of spectrophotometric methods for quantitative determination of caroverine in pharmaceutical pure and tablet formulations. Analytical Chemistry research. 2015;4:33-38.

6. A Raza and TM Ansari. Development and Validation of Novel Analytical Method for the Determination of Caroverine in Pharmaceutical Formulations. Austin J Anal Pharm Chem. 2015;2(1):1031.

7. P Job. Advanced Physicochemical Experiments (2nd ed.) Oliner and Boyd, Edinburgh.1964;54.

8. HA Benesi and J Hidelbrand. A Spectrophotometric Investigation of the Interaction of Iodine with Aromatic Hydrocarbons. J Am Chem Soc. 1949;71(8):2703-2707

9. AN Martin, J Swarbrick, A Cammarata. Physical Pharmacy. 3rd ed. Lee \& Febiger, Philadelphia, PA. 1969;371-374, 344-346.

10.ICH Guidance on Analytical Method Validation, [in:] Proc. Int.Convention on Quality for the Pharmaceutical Industry, Toronto, Canada. 2002. 